\title{
The Araucaria project. Precise physical parameters of the eclipsing binary IO Aquarii
}

\author{
D. Graczyk ${ }^{1,2}$, P. F. L. Maxted ${ }^{3}$, G. Pietrzyński ${ }^{4,2}$, B. Pilecki ${ }^{4,2}$, P. Konorski ${ }^{4}$, W. Gieren ${ }^{2,1}$, J. Storm ${ }^{5}$, A. Gallenne ${ }^{2}$, \\ R. I. Anderson ${ }^{6,7}$, K. Suchomska ${ }^{4}$, R. G. West ${ }^{8}$, D. Pollacco ${ }^{8}$, F. Faedi ${ }^{8}$, and G. Pojmański ${ }^{4}$
}

\author{
1 Millenium Institute of Astrophysics, Santiago 22, Chile \\ e-mail: darek@astro-udec.cl \\ 2 Universidad de Concepción, Departamento de Astronomía, Casilla 160-C, Concepción, Chile \\ 3 Astrophysics Group, Keele University, Staffordshire, ST5 5BG, UK \\ 4 Warsaw University Observatory, Al. Ujazdowskie 4, 00-478 Warsaw, Poland \\ 5 Leibniz-Institut für Astrophysik Potsdam, An der Sternwarte 16, 14482 Potsdam, Germany \\ ${ }^{6}$ Observatoire de Genève, Université de Genève, 51 Ch. des Maillettes, 1290 Sauverny, Switzerland \\ 7 Department of Physics and Astronomy, Johns Hopkins University, Baltimore, MD 21218, USA \\ ${ }^{8}$ Department of Physics, University of Warwick, Coventry CV4 7AL, UK
}

Received 29 March 2015 / Accepted 18 June 2015

\section{ABSTRACT}

\begin{abstract}
Aims. Our aim is to precisely measure the physical parameters of the eclipsing binary IO Aqr and derive a distance to this system by applying a surface brightness - colour relation. Our motivation is to combine these parameters with future precise distance determinations from the Gaia space mission to derive precise surface brightness - colour relations for stars.

Methods. We extensively used photometry from the Super-WASP and ASAS projects and precise radial velocities obtained from HARPS and CORALIE high-resolution spectra. We analysed light curves with the code JKTEBOP and radial velocity curves with the Wilson-Devinney program.

Results. We found that IO Aqr is a hierarchical triple system consisting of a double-lined short-period $(P=2.37 \mathrm{~d})$ spectroscopic binary and a low-luminosity and low-mass companion star orbiting the binary with a period of $\gtrsim 25000 \mathrm{~d}(~ \gtrsim 70 \mathrm{yr})$ on a very eccentric orbit. We derive high-precision (better than 1\%) physical parameters of the inner binary, which is composed of two slightly evolved main-sequence stars (F5 V-IV + F6 V-IV) with masses of $M_{1}=1.569 \pm 0.004$ and $M_{2}=1.655 \pm 0.004 M_{\odot}$ and radii $R_{1}=2.19 \pm 0.02$ and $R_{2}=2.49 \pm 0.02 R_{\odot}$. The companion is most probably a late K-type dwarf with mass $\approx 0.6 M_{\odot}$. The distance to the system resulting from applying a $(V-K)$ surface brightness - colour relation is $255 \pm 6$ (stat.) \pm 6 (sys.) pc, which agrees well with the HIPPARCOS value of $270_{-55}^{+91} \mathrm{pc}$, but is more precise by a factor of eight.
\end{abstract}

Key words. binaries: eclipsing - binaries: spectroscopic - stars: fundamental parameters - stars: distances - stars: solar-type

\section{Introduction}

Detached eclipsing binary stars are a very important source of precise stellar parameters, especially of absolute radii and masses. It has been proven that such parameters combined with empirical stellar surface brightness - colour (SBC) relations derived from interferometric measurements (e.g. Kervella et al. 2004; Challouf et al. 2014) result in accurate distance determinations even to extragalactic eclipsing binaries. Indeed, very accurate distances to the two Magellanic Clouds determined with the eclipsing binary method have recently been reported (Pietrzyński et al. 2013; Graczyk et al. 2014). In this method, the SBC relation is used as a tool for predicting the angular diameters of the system components (Lacy 1977). The distance is simply derived by comparing the absolute diameter of a component with its predicted angular diameter. The method involves simple geometric considerations and is therefore a powerful tool for measuring distances with a minimum of modelling assumptions.

However, the procedure may be reversed: using prior knowledge of the distance to a particular detached eclipsing binary, SBC empirical relations can be derived in a way completely independent of interferometry. This basic idea has long been known and was firstly used by Stebbins $(1910,1911)$ to derive the surface brightness of the two components of Algol and $\beta$ Aur. Within about $250 \mathrm{pc}$ of the Sun there are $\sim 100$ confirmed detached eclipsing binaries with parallaxes measured by the Hipparcos mission (e.g. Kruszewski \& Semeniuk 1999; Torres et al. 2010). However, about half of them still lack a detailed analysis of their physical parameters or any analysis at all. These systems comprise components covering a wide range of spectral types from $\mathrm{B} 1$ to $\mathrm{K} 0$ that is suitable for calibrating precise SBC relations in the corresponding colour ranges. The are several factors that influence the precision of SBC calibrations, most importantly, 1) the uncertainty on absolute dimensions, especially the radii; 2) trigonometric parallax errors; 3) intrinsic variability of the star; and 4) zero-point shifts between different photometric systems.

Recently, a few catalogues of precise absolute dimension determinations from eclipsing binaries have been published (Torres et al. 2010; Eker et al. 2014; Southworth 2014) that cover a wide range of temperatures. However, these catalogues show that for the purpose of SBC calibration, a precise analysis of new eclipsing binaries is needed. For example, Torres et al. (2010, see their Fig. 13) listed 15 components from nine systems (EI Cep, 
V422 Cyg, RZ Cha, GX Gem, BW Aqr, DM Vir, CD Tau, AI Phe, and V432 Aur) in a region of $T_{\text {eff }}-\log g$ plane covering a box of 6000-7000 K, 3.6-4.1 dex (F-type subgiants). Despite the number of systems with well-determined masses and radii in this region of parameter space, the calibration of the SBC for these stars is not very reliable because one system lacks a trigonometric parallax (V442 Cyg), three systems have very low quality trigonometric parallaxes (GX Gem, BW Aqr, and DM Vir), the HIPPARCos parallax of another system (AI Phe) is inconsistent with the value derived using the eclipsing binary method (Andersen et al. 1988), and one system is magnetically active with distortions to the light curve from star spots (V432 Aur). This leaves only five components in three non-active, detached eclipsing binaries with precise absolute dimension determinations within $250 \mathrm{pc}$ from the Sun, and with secure trigonometric parallaxes.

It is believed that parallax uncertainties from HIPPARCos are dominated by statistical errors - photon statistics (e.g. van Leeuwen 2007b). For F-type eclipsing binary with a parallax of 4 mas we expect an error of about 1 mas, or in other words, a relative precision of $25 \%$. To improve this, we could average results from many components over colour bins because the statistical uncertainty would decrease as $\sqrt{N}$, where $N$ is the number of components used in analysis.

The Gaia mission (Perryman et al. 2001) is expected to much improve the HIPPARCos distances by minimising statistical uncertainties, and for the F-type system mentioned before, the uncertainty of the parallax should be at most $0.5 \%$ (de Bruijne et al. 2015). The SBC calibration would benefit from such precise and accurate Gaia parallaxes obtained for a larger number of detached eclipsing binaries. The photometric distances derived for many of them would also be used as an independent check of Gaia results (if some significant systematic were to occur).

Inhomogeneous photometry causes difficulties with transformations between different photometric systems and zero-point shift uncertainties. The situation somewhat resembles problems occurring with the accurate determination of temperatures and angular diameters using the infrared flux method, where the main error comes from photometric calibration uncertainties (see discussion in Casagrande et al. 2014). Anticipating highly accurate parallaxes from Gaia (precision better than 1\%) and the standard precision of present-day radii determination (better then $2 \%$ ), the systematic error of future SBC calibration would be dominated by photometric and radiative properties of stellar atmosphere uncertainties (Andersen, priv. communication).

Here, we present a detailed analysis of IO Aqr (HD 196991, HIP 102041, $\left.\alpha_{2000}=20^{\mathrm{h}} 40^{\mathrm{m}} 45^{\mathrm{s}} .5, \delta_{2000}=00^{\circ} 56^{\prime} 21^{\prime \prime}\right)$, an eclipsing binary system composed of two mid-F-type stars located in the middle of the $T_{\text {eff }}-\log g$ plane of the region described above. Despite being relatively bright ( $V \sim 8.8 \mathrm{mag}$ ), this star was not recognised as an eclipsing binary until it was observed with HIPPARCOS and assigned its variable star name by Kazarovets et al. (1999). Time-series photometry is also available from the Wide-Angle Search for Planets (WASP; Pollacco et al. 2006). During our analysis we discovered that IO Aqr is a triple system, where a close inner binary has a low-mass companion star on a much wider orbit. The system was analysed before by Dimitrov et al. (2004). They derived a set of fundamental parameters for the binary, but did not detect any trace of the tertiary component. Although the companion complicates the interpretation of observations for IO Aqr, the companion is optically faint (less than $1 \%$ of the total light in $V$ ) and, being on a relatively wide orbit, exerts only long-term perturbations on the inner system, so we have been able to determine precise parameters for both stars in the eclipsing binary component of this hierarchical triple system.

\section{Observations}

\subsection{Photometry}

\subsubsection{ASAS}

The $V$-band photometry of IO Aqr, publicly available from the ASAS catalogue $^{1}$ (ACVS; Pojmański 2002), spans from 2001 March 27 to 2009 December 1 and contains 384 good-quality points (flagged "A" in the original data). This photometry was extended by 266 measurements from ASAS-North obtained in a second ASAS station on Haleakala, Hawaii. We also have access to previously unpublished I-band photometry from the ASAS and ASAS-North catalogues that spans from 1998 August 21 to 2009 June 4 and contains 1174 good points. In total we have four ASAS data sets, two in $V$ band and two in $I$ band.

\subsubsection{WASP}

IO Aqr (1SWASP J204045.47+005621.0) is one of several million bright stars ( $8 \lesssim V \lesssim 13$ ) that have been observed by WASP. The WASP survey is described in Pollacco et al. (2006) and Wilson et al. (2008). The survey obtains images of the night sky using two arrays of eight cameras, each equipped with a chargecoupled device (CCD), 200-mm f/1.8 lenses and a broad-band filter (400-700 nm). Two observations of each target field are obtained every $5-10$ min using two $30 \mathrm{~s}$ exposures. The data from this survey are automatically processed and analysed to identify stars with light curves that contain transit-like features that may indicate the presence of a planetary companion. Light curves are generated using synthetic aperture photometry with an aperture radius of 48 arcsec. The data for all the stars in each target field are processed using the SYSREM algorithm (Tamuz et al. 2005) to produce differential magnitudes that are partly corrected for systematic noise trends in the photometry. Data for this study were obtained between 2006 July 4 and 2010 October 6 .

\subsubsection{HIPPARCOS}

We downloaded the HIPPARCos photometry in $H_{\mathrm{P}}$ magnitudes from a public archive ${ }^{2}$. We removed four discrepant high points from the data set and finally had 87 photometric points in total.

\subsection{Spectroscopy}

\subsubsection{HARPS}

We obtained spectra of IO Aqr with the High Accuracy Radial velocity Planet Searcher (HARPS; Mayor et al. 2003) on the European Southern Observatory $3.6 \mathrm{~m}$ telescope in La Silla, Chile. IO Aqr is a bright target, therefore observations were generally obtained in marginal observing conditions or during twilight. Observations were obtained between 2009 October 10 and 2014 September 10. A total of 17 spectra were secured in high-efficiency ("EGGS") mode. The exposure times were typically $260 \mathrm{~s}$, resulting in an average signal-to-noise ratio $(\mathrm{S} / \mathrm{N})$ per pixel of $\sim 60$. All spectra were reduced on-site using the HARPS data reduction software (DRS).

\footnotetext{
1 http://www . astrouw . edu.pl/asas/?page=aasc

2 https://www.rssd.esa.int/index.php?

project=HIPPARCOS\&page=Epoch_Photometry
} 


\subsubsection{CORALIE}

Twenty spectra were obtained with the CORALIE spectrograph on the Swiss $1.2 \mathrm{~m}$ Euler telescope at La Silla observatory, Chile, between 2008 October 5 and 2010 November 1. The exposure times varied from $780 \mathrm{~s}$ to $1200 \mathrm{~s}$, giving a typical $\mathrm{S} / \mathrm{N}$ near $5500 \AA$ of 50 per pixel. The spectra were reduced using the automated reduction pipeline.

\section{Analysis}

\subsection{JKTEBOP}

We used JKTEBOP ${ }^{3}$ version 25 (Southworth et al. 2004a,b and references therein) to perform least-squares fits of the EBOP light-curve model (Popper \& Etzel 1981) to the various photometric data sets.

For the epoch of primary eclipse for each photometric data set, $T_{0}$, we used the time of mid-eclipse that was closest to the average time of observation for that data set. Other free parameters in the least-squares fit were a normalisation constant, the surface brightness ratio $J=S_{2} / S_{1}$, where $S_{1}$ and $S_{2}$ are the surface brightness of the primary and the secondary star, respectively; the sum of the radii relative to semi-major axis, $r_{1}+r_{2}=\left(R_{1}+R_{2}\right) / a$; the ratio of the radii, $k=r_{2} / r_{1}$; the orbital inclination, $i$ and the orbital period, $P_{\text {orb }}$. The mass ratio was fixed at the value derived from the spectroscopy described below. We consulted various tabulations of linear limb-darkening coefficients (van Hamme 1993; Díaz-Cordovés et al. 1995; Claret et al. 1995; Claret 2000) and then set the value of $x_{\mathrm{LD}}$ for both stars to be equal to a fixed average value from these tabulations. We performed independent least-squares fits to investigate the sensitivity of the other parameters to the assumed value of $x_{\mathrm{LD}}$ and accounted for this additional uncertainty when calculating the standard errors on these parameters. We assumed that the orbit is circular and fixed the gravity-darkening coefficients of both stars to 0.2 - this parameter has a negligible effect on the results.

The luminosity ratio and third-light contribution estimated from the analysis of the spectroscopic data were included as constraints in the least-squares fits. The relative weightings of the data were set equal to the root-mean-square (rms) residual of a preliminary fit to each data set. We used the residual permutation method to estimate robust standard errors on the free parameters. The results are given in Table 3, and the fits to the individual light curves are shown in Fig. 1. Further details of these fits are given below.

\subsubsection{WASP photometry}

We divided the WASP data into eight subsets, each subset comprising the data from one camera and one observing season. Outliers were identified using the residuals from a preliminary light curve model and were removed from the analysis. The number of data points used in the analysis and observation dates of each subset are given in Table 1. We identified between 6 and 25 nights in each subset of data where there was an offset between the data observed on that night and the rest of the data. We modified our version of JKTEBOP to include an offset for the data obtained on each of these nights as additional free parameters in the least-squares fit. The values and standard errors given in Table 3 are the weighted mean and standard error of the mean for the results from these eight least-squares fits. The times

\footnotetext{
3 www.astro.keele.ac.uk/ jkt/codes/jktebop.html
}

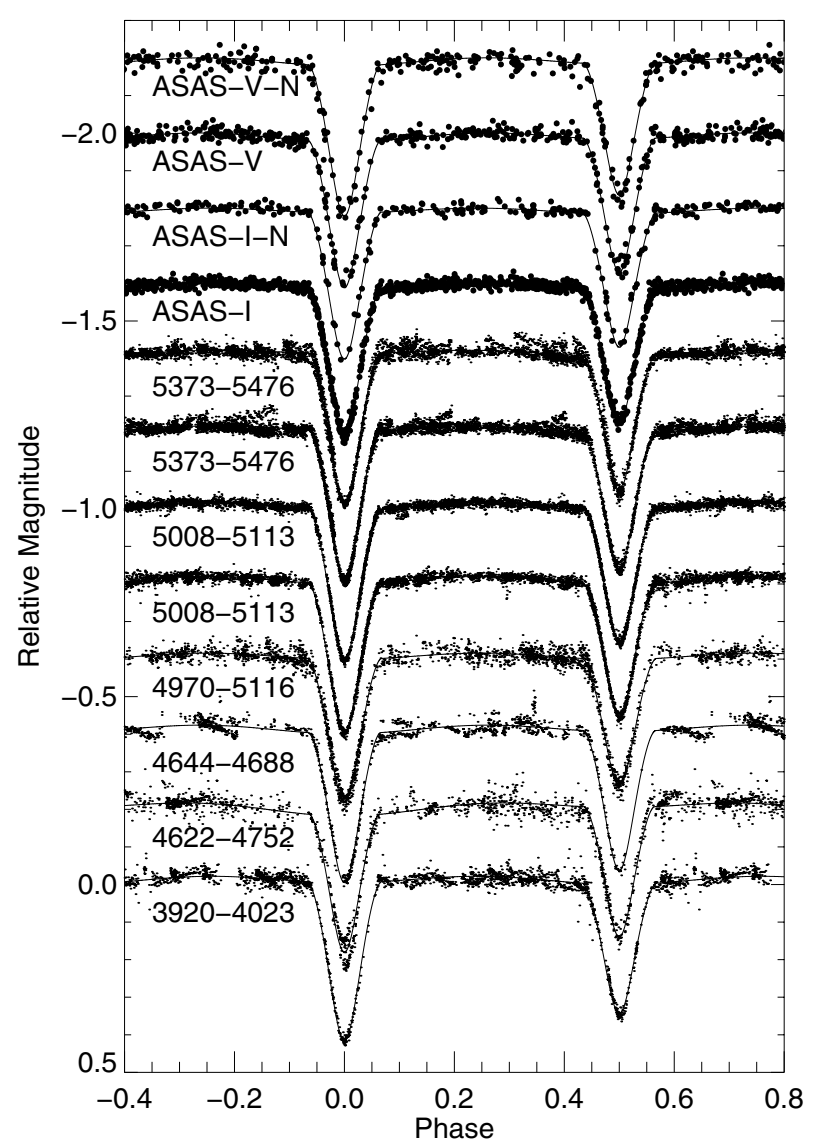

Fig. 1. From bottom to top: WASP, ASAS I-band, and ASAS $V$-band photometry of IO Aqr with JKTEBOP light-curve model fits. The numbers are the ranges of Julian dates for WASP data.

Table 1. Log of WASP photometry for IO Aqr.

\begin{tabular}{lrrr}
\hline \hline$t_{\text {start }}$ & $t_{\text {end }}$ & $N_{\text {ok }}$ & $N_{\text {block }}$ \\
\hline 3920 & 4024 & 1865 & 17 \\
4622 & 4753 & 1339 & 9 \\
4644 & 4689 & 1094 & 6 \\
4970 & 5117 & 2251 & 6 \\
5008 & 5114 & 3825 & 25 \\
5008 & 5114 & 4944 & 18 \\
5373 & 5477 & 5699 & 22 \\
5373 & 5477 & 4522 & 25 \\
\hline
\end{tabular}

Notes. The start and end dates of observation, $t_{\text {start }}$ and $t_{\text {end }}$, are JD-2 $450000 . N_{\text {ok }}$ is the number of data points used in this analysis, and $N_{\text {block }}$ is the number of nights of data that were fitted with an independent zero point in the least-squares fit.

of mid-eclipse derived from these least-squares fits are given in Table 2.

\subsubsection{ASAS photometry}

We fitted the four ASAS data sets independently. These data span a wide range of dates of observation with only one observation per night. The effect of the period variations discussed below on the parameters derived is negligible. To derive times of mid-primary eclipse for the period analysis, we divided the data into subsets, each covering approximately 200 nights. We then 
Table 2. Times of mid-primary eclipse for IO Aqr expressed in heliocentric Julian date.

\begin{tabular}{lrl}
\hline \hline Epoch & HJD & Source \\
\hline-2485 & $2448161.3007 \pm 0.0010$ & HIPPARCOS \\
-1248 & $2451090.6515 \pm 0.0008$ & ASAS-I \\
-1128 & $2451374.8234 \pm 0.0002$ & ASAS-I \\
-694 & $2452402.5822 \pm 0.0013$ & ASAS-I-N \\
-507 & $2452845.4146 \pm 0.0013$ & ASAS-I-N \\
-207 & $2453555.8503 \pm 0.0014$ & ASAS-I-N \\
-59 & $2453906.3304 \pm 0.0023$ & ASAS-I-N \\
272 & $2454690.1840 \pm 0.0008$ & ASAS-I-N \\
-779 & $2452201.2893 \pm 0.0059$ & ASAS-V \\
-657 & $2452490.1977 \pm 0.0025$ & ASAS-V \\
-494 & $2452876.1983 \pm 0.0009$ & ASAS-V \\
-193 & $2453589.0018 \pm 0.0016$ & ASAS-V \\
-70 & $2453880.2872 \pm 0.0024$ & ASAS-V \\
121 & $2454332.5974 \pm 0.0018$ & ASAS-V \\
269 & $2454683.0784 \pm 0.0010$ & ASAS-V \\
431 & $2455066.7165 \pm 0.0020$ & ASAS-V \\
-16 & $2454008.1642 \pm 0.0025$ & ASAS-V-N \\
94 & $2454268.6547 \pm 0.0042$ & ASAS-V-N \\
581 & $2455421.9382 \pm 0.0016$ & ASAS-V-N \\
740 & $2455798.4733 \pm 0.0011$ & ASAS-V-N \\
903 & $2456184.4783 \pm 0.0017$ & ASAS-V-N \\
-24 & $2453989.2174 \pm 0.0009$ & WASP \\
264 & $2454671.2380 \pm 0.0013$ & WASP \\
272 & $2454690.1845 \pm 0.0011$ & WASP \\
422 & $2455045.4056 \pm 0.0007$ & WASP \\
425 & $2455052.5095 \pm 0.0003$ & WASP \\
427 & $2455057.2459 \pm 0.0002$ & WASP \\
576 & $2455410.0988 \pm 0.0006$ & WASP \\
577 & $2455412.4671 \pm 0.0005$ & WASP \\
\hline & &
\end{tabular}

performed least-squares fits with only $T_{0}, J$, and a scaling factor as free parameters to derive the times of mid-primary eclipse given in Table 2.

\subsubsection{HIPPARCOS photometry}

We used HIPPARcos photometry to derive the single time of mid-primary eclipse given in Table 2 using the same method as we used for the ASAS data.

\subsection{Period variations}

We performed a least-squares fit to data given in Table 2 to derive the following linear ephemeris for the time of mid-primary eclipse in heliocentric Julian date:

HJD $(\mathrm{UTC})=2454046.0596(6)+2.3681172(8) \cdot E$.

The standard error on the final digit of each term is given in parentheses. The residuals from this ephemeris are shown in Fig. 2. It is clear that the period of this binary varies. Assuming that these changes are periodic, we can estimate their minimum period to be $>8000 \mathrm{~d}$. This is discussed further in Sect. 3.6.

\subsection{Radial velocities}

We used RaVeSpAn (Pilecki et al. 2012) to measure the radial velocities of both stars in the IO Aqr binary system using the broadening function (BF) formalism (Rucinski 1992, 1999). We used templates from the library of synthetic LTE spectra by Coelho et al. (2005). We chose templates to match the estimated effective temperature and gravity of the stars in the binary.

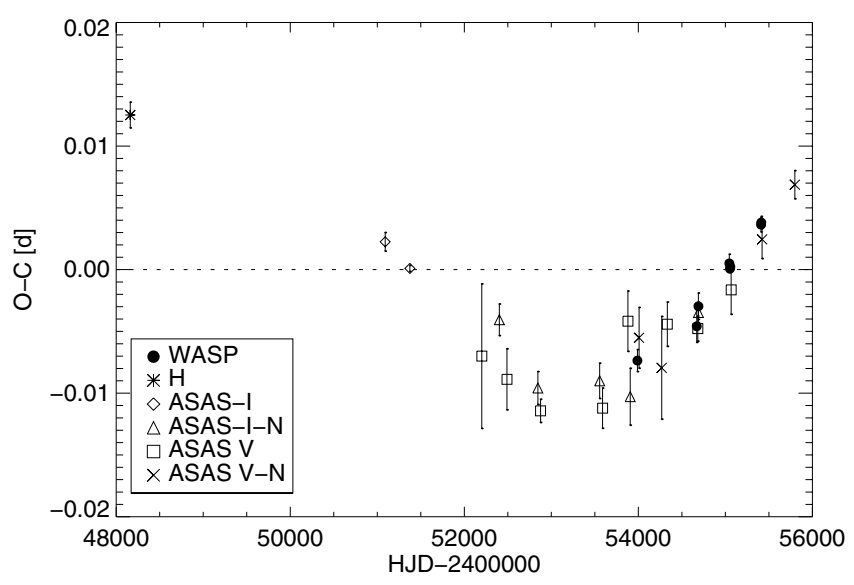

Fig. 2. Residuals from the best-fit linear ephemeris to our measured times of mid-primary eclipse.

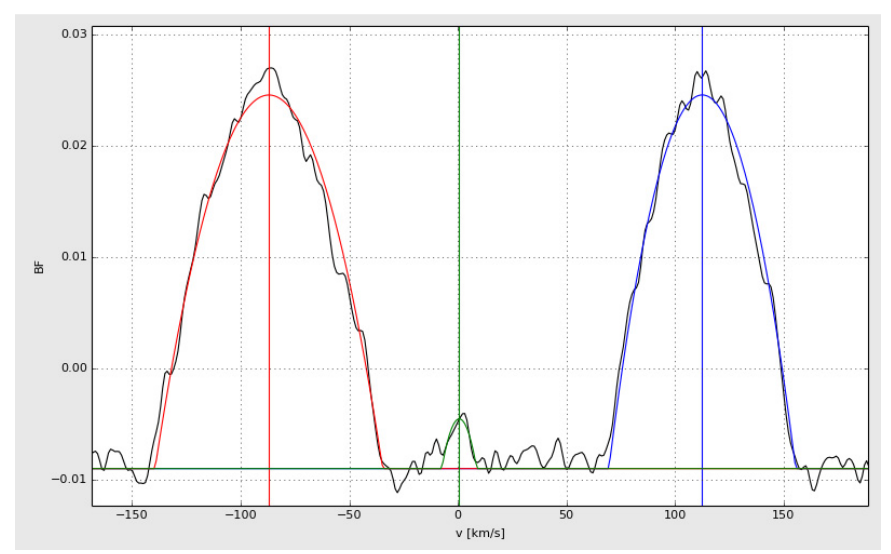

Fig. 3. Broadening function power diagram of IO Aqr's HARPS spectrum taken 2010 September 29 (JD = 2455468.5 ) during full astronomical night with the moon well below horizon. The low-temperature template $\left(T_{\text {eff }}=3950 \mathrm{~K}\right)$ was used to highlight the signal from the third body in the spectrum - the small bump in the middle. The paraboloid curves are rotationally broadened fits to the BF of the primary (right) and the secondary (left), and the continuous vertical lines are the resulting radial velocities of the three stars.

The abundance was assumed to be solar. We made preliminary radial velocity fits separately to the HARPS and CORALIE data. There are very small differences in the systemic velocities of both components, but as they are much smaller than dispersion of residuals, we neglected them. The resulting rms of the CORALIE radial velocities is about $30 \%$ larger than the rms of HARPS data. The line profiles of both stars are considerably broadened by the rotation of the stars. The projected equatorial rotation velocities of the primary and secondary components are $v_{1} \sin i=42.6 \pm 1.3 \mathrm{~km} \mathrm{~s}^{-1}$ and $v_{2} \sin i=52.3 \pm 1.1 \mathrm{~km}^{-1}$, respectively. The expected equatorial velocities for assuming synchronous rotation are $46.9 \mathrm{~km} \mathrm{~s}^{-1}$ and $53.6 \mathrm{~km} \mathrm{~s}^{-1}$ for the primary and the secondary, respectively, so it appears that rotation rates of both stars are very close to synchronous rotation. The line intensity ratio at $5500 \AA$ is 1.23 .

During this analysis, we noticed an additional faint feature in the BF near the radial velocity value $2 \mathrm{~km} \mathrm{~s}^{-1}$ in one of the spectra - see Fig. 3. We searched for this feature in the other spectra and confirmed its existence in every spectrum. The radial velocity of this feature slightly changes with a spread 
Table 3. Results of the JKTEBOP fit to the observed LCs.

\begin{tabular}{|c|c|c|c|c|c|c|}
\hline Parameter & WASP & ASAS-I & ASAS-I-N & ASAS-V & ASAS-V-N & Mean \\
\hline$T_{0}(\mathrm{HJD}-2450000)$ & - & $1396.13639(24)$ & $3589.0060(6)$ & $3589.0052(6)$ & $5315.3727(21)$ & - \\
\hline$P_{\text {orb }} \mathrm{d}$ & $2.368145(17)$ & $2.3681012(51)$ & $2.368121(2)$ & $2.368123(2)$ & $2.368136(3)$ & - \\
\hline$r_{1}+r_{2}$ & $0.4238(13)$ & $0.4256(31)$ & $0.4277(56)$ & $0.4192(48)$ & $0.4243(63)$ & $0.4244(14)$ \\
\hline$k=r_{2} / r_{1}$ & $1.1500(100)$ & $1.1462(39)$ & $1.1451(89)$ & $1.1551(78)$ & $1.1746(110)$ & $1.1478(36)$ \\
\hline$i(\operatorname{deg})$ & 81.44(8) & $81.47(18)$ & $81.43(26)$ & $81.74(22)$ & $81.45(28)$ & $81.47(7)$ \\
\hline$r_{1}$ & $0.1984(9)$ & $0.1938(17)$ & $0.1988(11)$ & $0.1945(25)$ & $0.1951(33)$ & $0.1976(18)$ \\
\hline$r_{2}$ & $0.2265(18)$ & $0.2273(15)$ & $0.2268(19)$ & $0.2247(24)$ & $0.2292(33)$ & $0.2268(16)$ \\
\hline$J=S_{2} / S_{1}$ & $0.908(6)$ & $0.929(5)$ & 0.911(4) & $0.918(12)$ & $0.888(18)$ & - \\
\hline$x_{\mathrm{LD}}$ & $=0.6$ & $=0.42(9)$ & $=0.42(9)$ & $=0.60(8)$ & $=0.60(8)$ & - \\
\hline$L_{3}(\%)$ & {$[1.0(5)]$} & {$[1.0(5)]$} & {$[1.0(5)]$} & {$[1.0(5)]$} & {$[1.0(5)]$} & - \\
\hline$L_{2} / L_{1}$ & {$[1.21(4)]$} & {$[1.23(3)]$} & {$[1.23(3)]$} & {$[1.21(3)]$} & {$[1.21(3)]$} & - \\
\hline rms (mag) & 0.014 & 0.009 & 0.011 & 0.014 & 0.018 & - \\
\hline
\end{tabular}

Notes. Values for the WASP data are the weighted means and standard errors from 8 subsets, as described in Sect. 3.1.1. Symbols are defined in Sect. 3.1. Values preceded by "=" are fixed parameters in the least-squares fit. Values in parentheses are standard errors on the final digits of the quantity shown. Values in square brackets were constrained within the limits indicated during the least-squares fit. Where errors are given on fixed parameters, independent least-squares fits were performed to calculate the contribution of the uncertainty shown to the standard errors quoted on the other parameters.

of about $2 \mathrm{~km} \mathrm{~s}^{-1}$. We cross-correlated our spectra with different templates and found that the maximum strength of the feature occurs for an assumed template temperature of $\sim 4000 \mathrm{~K}$ (assuming solar metallicity). The feature contributes about $1 \%$ of the total light at $\sim 6000 \AA$. Our radial velocity measurements for all three stars are given in Table 4.

The detection of period variations (Sect. 3.2) and third light in the spectra led us to the hypothesis that there is a third body in the system, probably a low-mass main-sequence star. This companion star probably is responsible for the additional signal in the spectrum and the period changes due to the light-time effect, which result from its orbital motion around the main binary.

\subsection{Interstellar extinction}

The SIMBAD/VizieR database quotes a few different spectral type estimates for IO Aqr: F5 V, F5 III, F6 V, and G0. This range is too broad to be useful for finding intrinsic average colours of the system. Instead, we used extinction maps (Schlegel et al. 1998) with recalibration by Schlafly \& Finkbeiner (2011) to determine the reddening in direction of IO Aqr. The total foreground reddening in this direction is $E(B-V)=0.070 \pm$ $0.001 \mathrm{mag}$. The reddening to IO Aqr is a fraction of this total. To estimate the reddening to IO Aqr, we assumed a simple axisymmetric model of an exponential disc for the distribution of dust within the Milky Way with scale and height lengths taken from Drimmel \& Spergel (2001) and assumed a distance to IO Aqr of $D=0.25 \mathrm{kpc}$ (see Sect. 4). For Galactic coordinates of IO Aqr of $(l, b)=\left(47^{\circ} .1,-23^{\circ} .6\right)$ and assuming a solar distance to the Milky Way centre of $R_{0}=8.3 \mathrm{kpc}$ (Gillessen et al. 2009), we obtain $E(B-V)_{\mathrm{IO} \text { Aqr }}=0.034 \mathrm{mag}$. Details of our procedure are given in Suchomska et al. (2015). Maxted et al. (2014) found the scatter of the reddening to individual stars around the value obtained from these reddening maps to be of 0.034 mag.

Additionally, we used a calibration between the equivalent width of the interstellar absorption sodium line NaI D1 and reddening by Munari \& Zwitter (1997). We averaged our measurements over several spectra of IO Aqr obtained in orbital quadratures between 2009 and 2014. The interstellar sodium line D1 has one sharp component of constant radial velocity of $-10.55 \mathrm{~km} \mathrm{~s}^{-1}$ The mean equivalent width of this line is $0.082 \AA$, which results in $E(B-V)=0.019 \mathrm{mag}$. The scatter of the $E(B-V)$ values for individual stars around
Table 4. RV measurements for IO Aqr.

\begin{tabular}{|c|c|c|c|c|}
\hline $\begin{array}{l}\text { BJD } \\
-2450000 \\
\end{array}$ & $\begin{array}{r}\mathrm{RV}_{1} \\
\left(\mathrm{~km} \mathrm{~s}^{-1}\right) \\
\end{array}$ & $\begin{array}{r}\mathrm{RV}_{2} \\
\left(\mathrm{~km} \mathrm{~s}^{-1}\right) \\
\end{array}$ & $\begin{array}{r}\mathrm{RV}_{3} \\
\left(\mathrm{~km} \mathrm{~s}^{-1}\right) \\
\end{array}$ & Spectr. \\
\hline 4745.49326 & $-84.34(20)$ & $99.64(23)$ & $3.0(1.1)$ & CORAL \\
\hline 4746.59946 & $117.50(20)$ & $-91.90(23)$ & $1.3(1.1)$ & CORAL \\
\hline 4746.70296 & $98.85(20)$ & $-74.51(23)$ & $4.6(1.1)$ & CORAL \\
\hline 4747.49989 & $-104.83(20)$ & $118.10(23)$ & $3.1(1.1)$ & CORAL \\
\hline 4747.63290 & $-110.13(20)$ & $123.30(23)$ & $3.0(1.1)$ & CORAL \\
\hline 5086.57856 & $-68.78(20)$ & $83.75(23)$ & $1.1(1.1)$ & CORAL \\
\hline 5086.59403 & $-64.90(20)$ & $80.74(23)$ & $2.0(1.1)$ & CORAL \\
\hline 5087.55728 & $123.67(20)$ & $-98.49(23)$ & $1.4(1.1)$ & CORAL \\
\hline 5087.66076 & $109.02(20)$ & $-85.24(23)$ & - & CORAL \\
\hline 5088.54850 & $-107.94(20)$ & $120.58(23)$ & $1.8(1.1)$ & CORAL \\
\hline 5088.63650 & $-110.38(20)$ & $122.96(23)$ & 2.2( & CORAL \\
\hline 5089.55659 & $103.59(20)$ & $-79.53(23)$ & $2.3(1.1)$ & CORAL \\
\hline 5089.64296 & $117.78(20)$ & $-93.71(23)$ & $0.9(1.1)$ & CORAL \\
\hline 5090.55337 & $-37.84(20)$ & $54.57(23)$ & - & CORAL \\
\hline 5090.62798 & $-58.25(20)$ & $74.19(23)$ & $0.3(1.1)$ & CORAL \\
\hline 5120.57639 & $129.38(15)$ & $-103.81(17)$ & $2.0(1.0)$ & HARPS \\
\hline 5447.66922 & $99.38(15)$ & $-75.39(17)$ & $2.4(1.0)$ & HARPS \\
\hline 5447.75236 & $79.77(15)$ & $-56.73(17)$ & $2.2(1.0)$ & HARPS \\
\hline 5449.50961 & $103.02(15)$ & $-79.17(17)$ & $1.3(1.0)$ & HARPS \\
\hline 5467.49889 & $-110.02(15)$ & $122.78(17)$ & $1.9(1.0)$ & HARPS \\
\hline 5468.50664 & $112.30(15)$ & $-88.17(17)$ & $1.5(1.0)$ & HARPS \\
\hline 5469.51563 & $-54.62(15)$ & 70.93(17) & $1.7(1.0)$ & HARPS \\
\hline 5479.51122 & $-101.29(15)$ & $115.13(17)$ & $2.0(1.0)$ & HARPS \\
\hline 5499.54423 & $128.36(20)$ & $-103.11(23)$ & & CORAL \\
\hline 439 & $127.18(20)$ & -101.40 & $.6(1.1)$ & CORAL \\
\hline 5500.53761 & $-101.57(20)$ & 114.93(23) & $.5(1.1)$ & CORAL \\
\hline 5500.57142 & $-105.01(20)$ & $118.33(23)$ & $1.3(1.0)$ & CORAL \\
\hline 5501.53207 & $85.99(20)$ & $-62.50(23)$ & 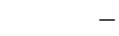 & CORAL \\
\hline 5536.52398 & $-68.88(15)$ & $84.30(17)$ & $0.7(1.0)$ & HARPS \\
\hline 6572.48783 & $108.47(15)$ & $-84.14(17)$ & $1.6(1.0)$ & HARPS \\
\hline 6579.49121 & $122.85(15)$ & $-97.65(17)$ & $1.9(1.0)$ & HARPS \\
\hline 6908.52162 & $129.63(15)$ & $-103.69(17)$ & $2.4(1.0)$ & HARPS \\
\hline 6908.60189 & $127.83(15)$ & $-102.24(17)$ & $1.2(1.0)$ & HARPS \\
\hline 6909.52328 & $-94.03(15)$ & $107.85(17)$ & $2.1(1.0)$ & HARPS \\
\hline 6909.58819 & $-102.62(15)$ & $116.23(17)$ & $3.0(1.0)$ & HARPS \\
\hline 6910.53338 & 76.02(15) & $-52.86(17)$ & $1.4(1.0)$ & HARPS \\
\hline 6910.65710 & $104.42(15)$ & $-80.37(17)$ & $1.2(1.0)$ & HARPS \\
\hline
\end{tabular}

Notes. Index "1" denotes the hotter star (primary); "2" is the cooler (secondary); and " 3 " is the tertiary component. Numbers in parenthesis give the uncertainty. BJD means the Barycentric Julian date and CORAL means the CORALIE spectrograph. 
Table 5. Observed magnitudes of the system IO Aqr.

\begin{tabular}{lccccc}
\hline \hline Band & & Ref. & \multicolumn{3}{c}{ IO Aqr } \\
& total(err.) & & primary & secondary & third body \\
\hline$B$ & $9.315(31)$ & 3 & 10.145 & 10.001 & $15.92^{a}$ \\
$V$ & $8.834(22)$ & 1 & 9.685 & 9.506 & $14.63^{a}$ \\
$R$ & $8.540(45)$ & 4,5 & 9.403 & 9.209 & $13.81^{a}$ \\
$I$ & $8.254(19)$ & 1 & 9.128 & 8.919 & $13.14^{b}$ \\
$J_{2 \text { MASS }}$ & $7.830(29)$ & 2 & 8.726 & 8.494 & $12.13^{b}$ \\
$K_{\text {2MASS }}$ & $7.555(33)$ & 2 & 8.474 & 8.222 & $11.36^{b}$ \\
\hline
\end{tabular}

Notes. ${ }^{(a)}$ Estimated from spectra. ${ }^{(b)}$ Assuming spectral type K6 V.

References. Source: 1 - this work (ASAS); 2 - Cutri et al. (2003); 3 - Høg et al. (2000) (Tycho-2); 4 - Pickles \& Depagne (2010); 5 Zacharias et al. (2004) (NOMAD).

the calibration of Munari et al. for low reddening values is approximately $0.04 \mathrm{mag}$. Both estimates point toward a small extinction in direction of IO Aqr. Finally, we assumed the extinction to be an average of both estimates: $E(B-V)=0.027 \pm$ $0.020 \mathrm{mag}$.

\subsection{Third-light characterisation}

We estimated the colour of the third light present in the spectrum using two spectral windows: a blue one ranging from $4000 \AA$ to $5000 \AA$ and a red one ranging from $6000 \AA$ to $6800 \AA$. These spectral windows approximately correspond to the Johnson $B$ and Johnson-Cousins $R$ photometric bands. Each window was used to calculate the integrated BF profile strength of every component visible in the spectra: $W_{1}, W_{2}, W_{3}$, where index 1 is for the primary, etc. The profiles were calculated using a template with $T_{\text {eff }}=6350 \mathrm{~K}, \log g=3.00$, and solar metallicity. We defined the third light as $l_{3}=W_{3} /\left(W_{1}+W_{2}+W_{3}\right)$. Averaging over several spectra, we obtained $l_{3}(B)=0.0023$ and $l_{3}(R)=0.0078$. From the observed magnitudes of IO Aqr in the $B$ and $R$ bands, we calculated the magnitudes of the third light in these bands (see Table 5). They correspond to an observed colour $(B-R)=2.11 \mathrm{mag}$ and de-reddened colour $(B-R)_{0}=2.05$. Using Table 5 of Pecaut \& Mamajek $(2013)^{4}$, we found that this colour corresponds to a main-sequence star of spectral type K6 V. Assuming a distance of $255 \mathrm{pc}$ (Sect. 4) and a reddening of $E(B-V)=0.027$ (Sect. 3.4) to IO Aqr, we calculated the $R$ absolute magnitude of the companion to be $M_{R}=6.87 \mathrm{mag}$, which corresponds to a spectral type between $\mathrm{K} 5 \mathrm{~V}$ and $\mathrm{K} 6 \mathrm{~V}$. Adding our estimate of the effective temperature given at the end of Sect. 3.3, we conclude that the companion has a spectral type $\mathrm{K} 6 \pm 1$ subclass. Table 5 gives the magnitude of the companion in different photometric bands assuming colours from Pecaut \& Mamajek (2013). The expected contribution of the third light in $K$ band is $\sim 3 \%$.

We found that the derived $(B-R)$ colour depends only slightly on the chosen template, being on average redder for templates with lower effective temperature. Assuming the same distance for IO Aqr and the third-light source, it is unlikely that the source is of spectral type later than M0 V because it would then be too weak to produce a significant BF signal. On the other hand, a star with spectral type earlier than $\mathrm{K} 4 \mathrm{~V}$ is also unlikely because it would produce a much stronger BF signal that would match a hotter template $(\sim 4600 \mathrm{~K})$.

\footnotetext{
4 http://www . pas. rochester.edu/ emamajek/ EEM_dwarf_UBVIJHK_colours_Teff.txt
}

\subsection{Analysis of combined light and radial velocity curves}

To perform this analysis we used version 2013 of the WilsonDevinney program (hereafter WD; Wilson \& Devinney 1971; Wilson 1979, 1990; van Hamme \& Wilson 2007) ${ }^{5}$. We cleaned the WASP light curve with $3 \sigma$ clipping and repeated this cleaning three times. Then we used every third WASP observation to form the final light curve used in the WD analysis. This yielded light curves containing 4730 points in WASP band, 650 points in $V$ band, 1174 points in $I$ band, and 87 points in $H_{\mathrm{p}}$ band.

We set $T_{1}=6475 \mathrm{~K}$ (see Sect. 3.6.1) and $[\mathrm{Fe} / \mathrm{H}]=0$. The grid size was set to $N=40$, and standard albedo and gravity brightening for convective stellar atmospheres were chosen. The stellar atmosphere option was used, radial velocity tidal corrections were automatically applied, and no flux-leveldependent weighting was used. We assumed a circular orbit and synchronous rotation for the two components. Logarithmic limbdarkening law were used (Klinglesmith \& Sobieski 1970). The starting point for the parameters of the binary system were based on the solutions from the JKTEBOP analysis augmented by dynamical parameters from the preliminary solution obtained with RaVeSpAn, but we also accounted for the influence of the third body on the orbit, as we describe below.

\subsubsection{Effective temperature estimates}

To estimate the effective temperatures of the eclipsing components, we used a number of $(V-K)$ and $(V-I)$ colour temperature calibrations (Ramírez \& Meléndez 2005; González Hernández \& Bonifacio 2009; di Benedetto 1998; Casagrande et al. 2010; Masana et al. 2006; Houdashelt et al. 2000). The intrinsic colours of the two components were calculated from observed magnitudes, estimated reddening, flux ratios derived from the WD model, and from the third-light contribution. As a source for $K$-band photometry in 2MASS (Cutri et al. 2003) (see Table 5), we used appropriate colour transformations for each calibration. Because flux ratios from the WD model depend on the input temperatures, we iterated the estimation of temperatures a few times. The resulting temperatures are averages from all used calibrations. For the primary and secondary they are $6475 \pm 85 \mathrm{~K}$ and $6331 \pm 69 \mathrm{~K}$, respectively, with errors calculated as the standard deviation. When the reddening and zero-point uncertainties of the photometry are added, the total errors of the temperature determination are $138 \mathrm{~K}$ and $125 \mathrm{~K}$ for the primary and the secondary, respectively.

\subsubsection{Analysis including the third body}

We assumed that the changes in orbital period are caused entirely by the light-time effect (Woltjer 1922; Irwin 1959; van Hamme \& Wilson 2007). Changes in the orbital period of the inner binary due to gravitational interaction between the three bodies are expected to be at least two orders of magnitude smaller than the variation seen in Fig. 2 (Rappaport et al. 2013, their Eq. (10)). A preliminary analysis of our radial velocities showed that the systemic velocity is $\gamma \approx 10 \mathrm{~km} \mathrm{~s}^{-1}$. Dimitrov et al. (2004) reported a much lower systemic velocity of $-2 \mathrm{~km} \mathrm{~s}^{-1}$ from their observations taken in Rozen Observatory with the $2.0 \mathrm{~m}$ RCC telescope, which is equipped with a Coudé spectrograph. Their spectra were taken in 2001, so there is a difference of about nine years to our spectra. We initially interpreted the change of the systemic velocity as a sign of interaction between the binary and the third

ftp://ftp.astro.ufl.edu/pub/wilson/lcdc2013/ 
body. However, we quickly realized that the magnitude of the systemic velocity change $\left(\sim 12 \mathrm{~km} \mathrm{~s}^{-1}\right)$ is much too large: if this were real, we would expect much larger $\mathrm{O}-\mathrm{C}$ residuals from the linear ephemeris given in Sect. 3.2. We concluded that there is a large systematic zero-point offset in velocities derived from our spectra and the Rozen spectra, and because their radial velocities are on average of lower accuracy than ours, we dropped them from our analysis.

In the analysis we adjusted the following parameters describing the inner binary of IO Aqr: the binary orbital period $P$, the zero-epoch of the primary minimum $\mathrm{HJD}_{0}$, the systemic velocity $\gamma$, the temperature of the secondary $T_{2}$, the orbital inclination $i$, the two surface potentials $\Omega_{1}$ and $\Omega_{2}$, the semi-major axis $a$, the mass ratio $q$, and the primary star luminosity $L_{1}$ in each photometric band.

The $\mathrm{O}-\mathrm{C}$ diagram shows that our eclipse timing and radial velocity data probably cover only a small fraction of the orbital period of the third body, $P_{3}$. In this case, the third body orbital parameters are not uniquely determined. A striking feature of our radial velocities is the lack of significant changes in the systemic velocity of the inner binary or the mean radial velocity of the third body. Moreover, the shape of the $\mathrm{O}-\mathrm{C}$ diagram suggests a highly eccentric and long-period third-body orbit. It was also clear from our trial solutions that the orbital period $P_{3}$ and the eccentricity $e_{3}$ strongly correlate, as is the case for the longitude of periastron $\omega_{3}$ and the time of the upper spectroscopic conjunction $T_{03}$. For this reason, we decided to find solutions within a grid of possible periods $P_{3}$. The additional fitted parameters were those describing the third-body orbit: eccentricity $e_{3}$, longitude of the periastron $\omega_{3}$, semi-major axis $a_{3}$, and the moment of the upper spectroscopic conjunction $T_{03}$. We also assumed that the outer orbit is seen edge-on $\left(i_{3}=90 \mathrm{deg}\right)$.

We decided to divide the third-body parameters into three groups $-\left\{e_{3}, T_{03}\right\},\left\{\omega_{3}\right\}$, and $\left\{a_{3}, T_{03}\right\}$. We iteratively fitted these three parameter groups with the parameters of the inner binary $\left(P, \mathrm{HJD}_{0}, \gamma, T_{2}, i, \Omega_{1}, \Omega_{2}, a, q\right.$, and $\left.L_{1}\right)$ until we achieved no improvement in $\chi^{2}$ for a given value of $P_{3}$. We repeated this procedure for many orbital periods $P_{3}$ within a range of from 8000 to 50000 days. We could not find any acceptable third-body solutions for periods shorter than about 25000 days. The reason is that the radial velocity difference between the inner binary systemic velocity and the radial velocity of the third body $\left(\Delta V_{\mathrm{r}} \approx 8 \mathrm{~km} \mathrm{~s}^{-1}\right)$ causes a tension with the shape of the $\mathrm{O}-\mathrm{C}$ curve. The tension is only relaxed for $P_{3}>25000$ days. Periods longer than $50000 \mathrm{~d}$ require a very high eccentricity of the outer orbit $\left(e_{3}>0.85\right)$. In all cases the inferred minimum mass of the third body is about $0.6 M_{\odot}$. The specific solution for $P_{3}=30000$ days is reported in Table 6. Figure 4 presents the fit to the radial velocity data given in Table 4 assuming a constant orbital period during the time span of our spectroscopic data.

Figure 5 presents the fit to the $\mathrm{O}-\mathrm{C}$ residuals of observed minima times from Table 2 and the expected radial velocity changes of the inner binary mass centre of IO Aqr and the third body. To calculate the binary centre-of-mass velocities $V_{1+2}$, we used radial velocities from Table 4 and the equation

$V_{1+2}=\frac{V_{1}+q V_{2}}{1+q}$

where $V_{1,2}$ denotes radial velocities for the primary and the secondary, respectively, and $q$ is the mass ratio. The asymmetric minimum in the $\mathrm{O}-\mathrm{C}$ diagram coincides with the periastron passage of the third body near JD 2452600 . The O-C diagram shows that our final solution for the third body orbit does not fit the HIPPARCos data. Forcing a good fit for this one outlier results
Table 6. Results of the final WD analysis including the third body.

\begin{tabular}{|c|c|c|}
\hline Parameter & Primary & Secondary \\
\hline$P(\mathrm{~d})$ & \multicolumn{2}{|c|}{$2.3681275(33)$} \\
\hline $\mathrm{HJD}_{0}(\mathrm{~d})$ & \multicolumn{2}{|c|}{$2454046.0586(36)$} \\
\hline$a\left(R_{\odot}\right)$ & \multicolumn{2}{|c|}{$11.048(7)$} \\
\hline$q$ & \multicolumn{2}{|c|}{$1.0545(14)$} \\
\hline$i(\operatorname{deg})$ & \multicolumn{2}{|c|}{$81.56(6)$} \\
\hline$\Omega$ & $6.124(32)$ & $5.681(31)$ \\
\hline$r_{\text {mean }}$ & $0.1983(13)$ & $0.2257(15)$ \\
\hline$\gamma\left(\mathrm{km} \mathrm{s}^{-1}\right)$ & \multicolumn{2}{|c|}{$8.45(11)$} \\
\hline$T_{\text {eff }}(\mathrm{K})$ & $6475^{a}$ & $6336(6)$ \\
\hline$K\left(\mathrm{~km} \mathrm{~s}^{-1}\right)$ & $119.80(10)$ & $113.61(11)$ \\
\hline$L_{2} / L_{1}($ HIPPARCOS $)$ & \multicolumn{2}{|c|}{$1.165(15)$} \\
\hline$L_{2} / L_{1}(\mathrm{WASP})$ & \multicolumn{2}{|c|}{$1.176(14)$} \\
\hline$L_{2} / L_{1}(\mathrm{ASAS}-\mathrm{V})$ & \multicolumn{2}{|c|}{$1.178(14)$} \\
\hline$L_{2} / L_{1}($ ASAS-I $)$ & \multicolumn{2}{|c|}{$1.213(13)$} \\
\hline$l_{3}($ HIPPARCOS $)$ & \multicolumn{2}{|c|}{$0.0048^{a}$} \\
\hline$l_{3}(\mathrm{WASP})$ & \multicolumn{2}{|c|}{$0.0048^{a}$} \\
\hline$l_{3}(\mathrm{ASAS}-\mathrm{V})$ & \multicolumn{2}{|c|}{$0.0048^{a}$} \\
\hline$l_{3}(\mathrm{ASAS}-\mathrm{I})$ & \multicolumn{2}{|c|}{$0.0111^{a}$} \\
\hline$e_{3}$ & \multicolumn{2}{|c|}{$0.775(76)$} \\
\hline$\omega_{3}(\operatorname{deg})$ & \multicolumn{2}{|c|}{$227(16)$} \\
\hline$a_{3} \sin i_{3}\left(R_{\odot}\right)$ & \multicolumn{2}{|c|}{ 6354(30) } \\
\hline$T_{03}(\mathrm{~d})$ & \multicolumn{2}{|c|}{$2449611(584)$} \\
\hline$P_{3}(\mathrm{~d})$ & \multicolumn{2}{|c|}{$30000^{a}$} \\
\hline$q_{3}=M_{3} \sin i_{3} /\left(M_{1}+M_{2}\right)$ & \multicolumn{2}{|c|}{0.186} \\
\hline RV (HARPS) rms $\left(\mathrm{m} \mathrm{s}^{-1}\right)$ & 169 & 179 \\
\hline $\mathrm{RV}$ (CORALIE) $\mathrm{rms}\left(\mathrm{m} \mathrm{s}^{-1}\right)$ & 209 & 254 \\
\hline HIPPARCOS rms (mmag) & \multicolumn{2}{|c|}{14.6} \\
\hline WASP rms (mmag) & \multicolumn{2}{|c|}{7.1} \\
\hline ASAS-V rms (mmag) & \multicolumn{2}{|c|}{13.4} \\
\hline ASAS-I rms (mmag) & \multicolumn{2}{|c|}{8.4} \\
\hline$\chi^{2} /$ d.o.f. & \multicolumn{2}{|c|}{1.02} \\
\hline
\end{tabular}

Notes. ${ }^{(a)}$ Fixed value.

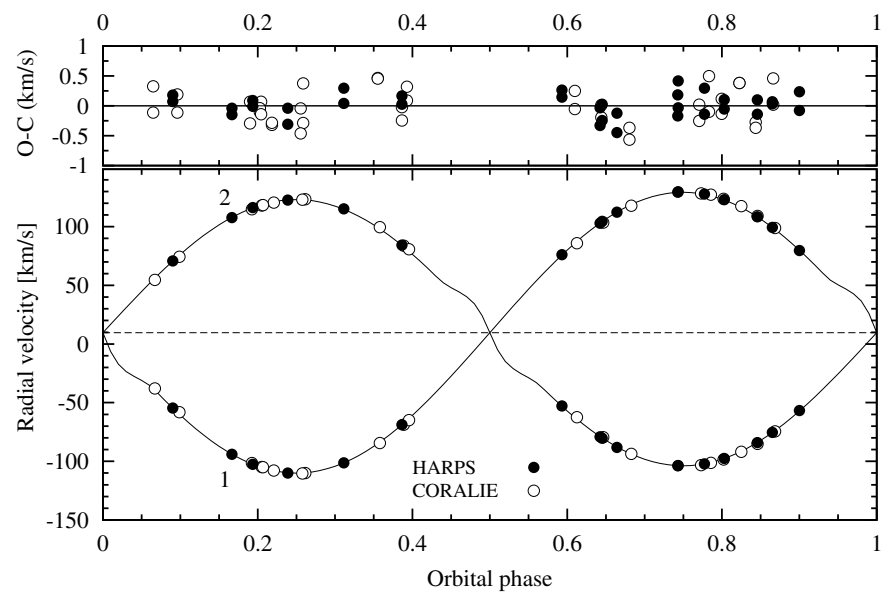

Fig. 4. Radial velocity solution of IO Aqr. The lower panel shows the model predictions with continous lines from the WD code calculated for a constant orbital period of $P=2.368137 \mathrm{~d}$. Overplotted are radial velocities for the primary (denoted 1) and the secondary (denoted 2). The dotted line corresponds to the systemic velocity of $9.66 \mathrm{~km} \mathrm{~s}^{-1}$ corresponding to a mean epoch of CORALIE and HARPS spectra. The upper panel shows residuals from the model fit.

in stronger binary centre-of-mass radial velocity changes and produces significant residua in the radial velocities. A possible explanation is that we underestimated the error on HIPPARCos minimum timing. It is also clear that we only cover a relatively 

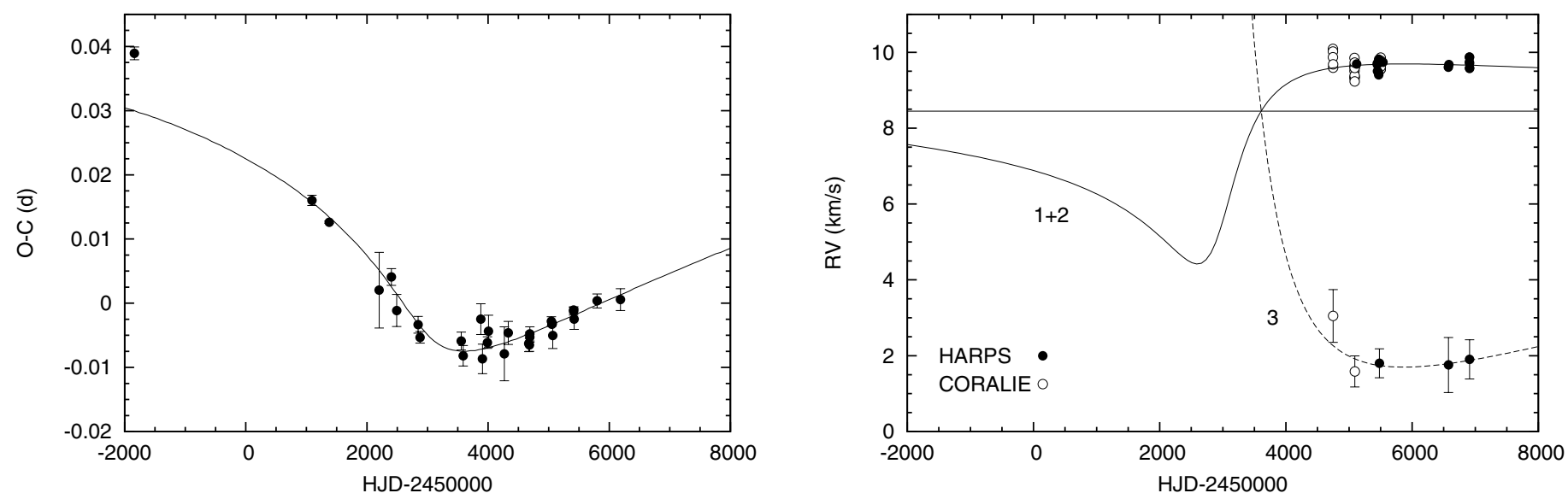

Fig. 5. Left panel: O-C residuals of the observed times of primary minimum of IO Aqr assuming the linear ephemeris from Table 6 and predicted $\mathrm{O}-\mathrm{C}$ caused by the light-time effect from our third-body solution - continous line. Right panel: predicted radial velocity changes of the binary mass centre (continuous line denoted 1+2) and the third body (dashed line denoted 3 ). The horizontal line corresponds to the heliocentric frame systemic velocity of the whole triple system. The individual binary mass centre velocities from HARPS are marked by filled circles and CORALIE velocities by open circles. The velocities of the third body were binned into seasonal means.

Table 7. Physical parameters of IO Aqr components.

\begin{tabular}{lccc}
\hline \hline Parameter & Primary & Secondary & Tertiary \\
\hline Spectral type & F5 V-IV & F6 V-IV & $\sim$ K6 V \\
$M\left(M_{\odot}\right)$ & $1.569(4)$ & $1.655(4)$ & $0.6(1)$ \\
$R\left(R_{\odot}\right)$ & $2.191(15)$ & $2.493(17)$ & - \\
$\log g(\mathrm{cgs})$ & $3.952(6)$ & $3.863(6)$ & - \\
$T_{\text {eff }}(\mathrm{K})$ & $6475(138)$ & $6336(125)$ & $\sim 4000$ \\
$L\left(L_{\odot}\right)$ & $7.59(55)$ & $9.00(65)$ & - \\
$M_{V}(\mathrm{mag})$ & $2.57(8)$ & $2.39(8)$ & $\sim 7.7$ \\
$\mathrm{BC} C_{V}(\mathrm{mag})$ & -0.021 & -0.030 & - \\
\hline Distance $(\mathrm{pc})$ & & $255(9)$ & \\
Distance modulus (mag) & & $7.030(72)$ & \\
Parallax (mas) & & $3.93(13)$ & \\
$E(B-V)(\mathrm{mag})$ & & $0.027(20)$ & \\
\hline
\end{tabular}

small part of the whole third-body orbit, and much room for improvement probably still exists.

\subsection{Physical parameters}

The physical parameters of the components of IO Aqr are presented in Table 7. Our spectral type estimate is based on the derived effective temperatures and the calibration by Pecaut $\&$ Mamajek (2013); it is consistent with the spectral classification given by Houk \& Swift (1999). The mean radii given are the radius of a sphere with the same volume as the star. The distortions from a perfect sphere defined as $\left(r_{\text {point }}-r_{\text {pole }}\right) / r_{\text {mean }}$ are $2.3 \%$ and $3.2 \%$ for the primary and the secondary star, respectively. Errors on luminosities were calculated from uncertainties of the distance estimate and the reddening and bolometric corrections used. The bolometric corrections reported in Table 7 were taken from Casagrande et al. (2010). The fractional precision of derived masses is $0.25 \%$ and $0.24 \%$ for the primary and the secondary, respectively.

Dimitrov et al. (2004) reported two possible solutions for absolute parameters of IO Aqr denoted as A and B. It becomes evident that our solution lies somewhere between these solutions. However, we were able to significantly refine the parameters of the system. The radii of the two stars are too large for their mass or for their temperature if we were to assume

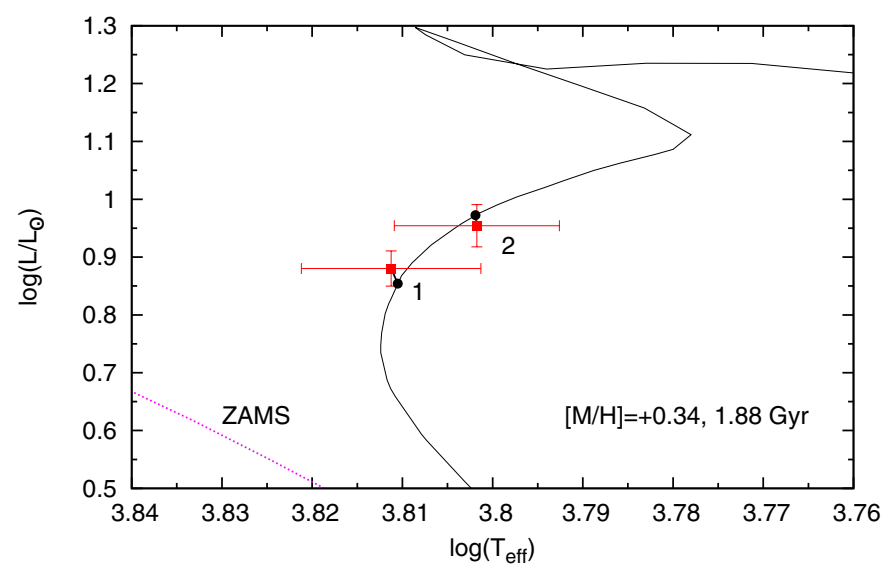

Fig. 6. H-R diagram with the primary (1) and the secondary (2) components of IO Aqr marked. Overplotted is the PARSEC isochrone that provides the best match to $M, L$, and $T_{\text {eff }}$ of the two stars, corresponding to an age of $1.88 \mathrm{Gyr}$. Dots on the isochrone correspond to the position of the best fit. The zero-age main-sequence (ZAMS) is also shown.

that they are main-sequence stars, as was also pointed out by Dimitrov et al. (2004). We compared our results with stellar isochrones from the Padova and Trieste Stellar Evolutionary Code (PARSEC; Bressan et al. 2012) ${ }^{6}$. We fitted masses, luminosities, and temperatures of the components. As we have no confident metallicity estimate for this star (the SIMBAD database quotes some estimates made under the assumption that IO Aqr is a single star), we also fitted this parameter. The position of the stars in the Hertzsprung-Russell (H-R) diagram and their measured masses are only matched using isochrones for metallicities $[\mathrm{M} / \mathrm{H}] \approx+0.3-$ see Fig. 6 . The estimated age of the system is $1.88 \pm 0.10$ Gyr. We confirm the finding of Dimitrov et al. (2004) that the two stars are still before the turn-off point in their evolution.

Our absolute parameters do not depend on the orbital solution for the third body. The third body only affects the timing of the eclipses and, to a lesser extent, the estimates of the distance and effective temperatures. Our solutions without the

6 http://stev.oapd.inaf.it/cgi-bin/cmd 
third body (Sect. 3.1.1) yield model parameters that are consistent with those reported in Table 6. The high precision of the derived physical parameters makes IO Aqr another eclipsing binary star on a list of systems that have a precision of the absolute dimension determination better than $3 \%$ (e.g. Torres et al. 2010).

\section{Distance and space velocity}

The distance to the system was derived using two calibrations of visual surface brightness versus $(V-K)$ colour relations (Kervella et al. 2004; di Benedetto 2005) based on measurements of the interferometric stellar angular diameters. The 2MASS $K$-band magnitude was tranformed onto the Johnson photometric system using transformation equations from Bessell \& Brett (1988) and Carpenter $(2001)^{7}$. The magnitudes were de-reddened with $E(B-V)=0.027 \mathrm{mag}$. To derive individual $K$-band magnitudes of the components, we used the $K$-band light ratio extrapolated from the WD model $l_{21}(K)=1.262$ and the thirdlight contribution $l_{3}(K)=0.030$. The resulting de-reddened Johnson $V$-band and $K$-band magnitudes for the two stars are $V_{1}=9.601 \mathrm{mag}, V_{2}=9.422 \mathrm{mag}$, and $K_{1}=8.505 \mathrm{mag}, K_{2}=$ $8.252 \mathrm{mag}$. The distance to IO Aqr derived from the SBC relations reported by Kervella et al. (2004) and di Benedetto (2005) are $257.2 \mathrm{pc}$ and $252.2 \mathrm{pc}$, respectively. The resulting average distance is $255 \mathrm{pc}$, corresponding to a distance modulus $m-M=7.030 \mathrm{mag}$.

The systematic errors contribute as follows: the uncertainty of the empirical calibrations of the surface brightness (0.040 mag error in distance modulus), the uncertainty of the extinction law ( $0.005 \mathrm{mag})$, the metallicity dependence of the surface brightness relations $(0.004 \mathrm{mag})$, and the zero-point errors of $V$ - and $K$-band photometry ( $0.030 \mathrm{mag}$ in total). When we combine the contributions in quadrature, we have a total systematic error of $0.051 \mathrm{mag}$. The total statistical error comes from the uncertainty in the semi-major axis $(0.005 \mathrm{mag})$, the uncertainty of the sum of fractional radii $r_{1}+r_{2}(0.010 \mathrm{mag})$, the uncertainty of the third light $(0.022 \mathrm{mag})$, the errors of $V$ - and $K$-band magnitudes ( 0.007 and $0.033 \mathrm{mag}$, respectively), the reddening uncertainty $(0.015 \mathrm{mag})$, the error of the mean of the two calibrations (0.021 mag), and from separating the magnitudes $(0.002 \mathrm{mag})$. When we combine these uncertainties in quadrature, we have a total statistical error of $0.049 \mathrm{mag}$. When the errors in distance modulus are translated into errors in parsecs, the final result is $255 \pm 6$ (stat.) \pm 6 (syst.) pc. The distance corresponds to a parallax of $3.93 \pm 0.13$ mas (total error).

The important consistency check of model parameters we derived (especially their radii and luminosity ratio in different bands) is the distance to each of the system components returned by our procedure. In our case, the distance moduli of the two stars agree excellently well: they are different by less than $0.001 \mathrm{mag}$ for each of the surface brightness calibration used.

The parallax to the binary was determined by the HIPPARCOS satellite mission. The first published full data reduction (Perryman et al. 1997) gave $5.42 \pm 1.46$ mas. Subsequently, a new data reduction (van Leeuwen 2007a,b) reported a distinctly smaller parallax of $3.71 \pm 0.94$ mas. Dimitrov et al. (2004) used an eclipsing binary bolometric flux scaling method and derived a distance to IO Aqr of $256 \mathrm{pc}$, corresponding to a parallax of 3.91 mas. However, they neither included any third light in their considerations nor gave a distance error estimate. Both of

\footnotetext{
http://www . astro. caltech. edu/ jmc/2mass/v3/ transformations/
}

the latter distance determinations agree very well within the uncertainties with our derived distance.

The proper motion of IO Aqr given by van Leeuwen (2007b) is $\left(\mu_{\alpha} \cos \delta, \mu_{\delta}\right)=(-4.59 \pm 1.00,-22.42 \pm 0.84) \mathrm{mas} \mathrm{yr}^{-1}$. That proper motion expressed in Galactic coordinates is $\left(\mu_{l} \cos b, \mu_{b}\right)=(-21.7 \pm 1.2,-7.4 \pm 0.4) \mathrm{mas} \mathrm{yr}^{-1}$ using the transformations given by Poleski (2013). The transverse velocity in Galactic coordinates is $(-26.2 \pm 1.5,-8.9 \pm 0.5) \mathrm{km} \mathrm{s}^{-1}$. Heliocentric Galactic space velocity components, that is, not corrected for solar peculiar motion, were calculated using the equations given by Johnson \& Soderblom (1987), and we obtained $(u, v, w)=(21.8 \pm 0.6,-14.9 \pm 1.6,-11.5 \pm 0.8) \mathrm{km} \mathrm{s}^{-1}$. The values and uncertainties given do not take into account possible systematic errors caused by a long-term proper motion drift of the inner binary induced by the third body. At the moment, we have insufficient information to correct for this effect.

\section{Final remarks}

With the high-precision data now available, we have been able to show that IO Aqr is a hierarchical triple star system with a lowluminosity and low-mass companion on a wide eccentric orbit. For the purpose of accurate SBC calibration, pure eclipsing binaries (i.e. without stellar companions) are preferred. However, when the physical properties, especially the luminosity, of a tertiary companion are well determined or/and the flux of the companion in optical and NIR is negligible, a given eclipsing binary can be used safely. A criterion is the degree of calibration precision we wish to obtain: $1 \%$ of precision demands that the tertiary contribution in $V$ and $K$ bands should be at most comparable with this limit. The companion of IO Aqr is faint enough in the optical to be ignored, and its estimated $K$-band contribution is still small, so that it has a negligible effect on the calculated angular diameters of the IO Aqr components. Using the SBC calibration of di Benedetto (2005), the predicted angular diameters are $0.081 \pm 0.002$ mas and $0.092 \pm 0.002$ mas for the primary and the secondary, respectively.

We derived high-precision absolute dimensions of IO Aqr that are among the most precise parameters of F-type stars published to date. Nonetheless, our temperature determination (precision 2\%) can be improved by using dedicated Strömgren photometry or by a detailed atmospheric analysis of the separated spectra. We leave this to future work on IO Aqr.

Acknowledgements. We extensively used the SIMBAD/Vizier database in our research. We also used the uncertainties python package. We would like to thank the referee D. Pourbaix for his constructive comments on the paper and the staff of the ESO La Silla observatory for their support during the observations. We also thank W. van Hamme for his valuable comments about modelling of a third body with the Wilson-Devinney code. We (D.G., W.G., G.P.) gratefully acknowledge financial support for this work from the BASAL Centro de Astrofisica y Tecnologias Afines (CATA) PFB-06/2007, and from the Millenium Institute of Astrophysics (MAS) of the Iniciativa Cientifica Milenio del Ministerio de Economia, Fomento y Turismo de Chile, project IC120009. A.G. acknowledges support from FONDECYT grant 3130361. We (D.G., B.P., G.P., P.K., K.S.) gratefully acknowledge financial support for this work from the Polish National Science Center grant MAESTRO 2012/06/A/ST9/00269, the TEAM subsidy from the Foundation for Polish Science (FNP) and NCN grant DEC-2011/03/B/ST9/02573. R.I.A. acknowledges funding from the Swiss National Science Foundation.

\section{References}

Andersen, J., Clausen, J. V., Gustafsson, B., et al. 1988, A\&A, 196, 128

Bessell, M. S., \& Brett, J. M. 1988, PASP, 100, 1134

Bressan, A., Marigo, P., Girardi, L., et al. 2012, MNRAS, 427, 127

Carpenter, J. M. 2001, AJ, 121, 2851 
Casagrande, L., Ramirez, I., Meléndez, J., Bessell, M., \& Asplund, M. 2010, A\&A, 512, A54

Casagrande, L., Portinari, L., Glass, I. S., et al. 2014, MNRAS, 439, 2060

Challouf, M., Nardetto, N., Mourard, D., et al. 2014, A\&A, 570, A104

Claret, A. 2000, A\&A, 363, 1081

Claret, A., Diaz-Cordoves, J., \& Gimenez, A. 1995, A\&AS, 114, 247

Coelho, P., Barbuy, B., Meléndez, J., Schiavon, R. P., \& Castilho, B. V. 2005, A\&A, 443, 735

Cutri, R. M., Skrutskie, M. F., van Dyk, S., et al. 2003, VizieR, Online Data Catalogue: II/246

de Bruijne, J. H. J., Rygl, K. L. J., \& Antoja, T. 2015, ArXiv e-prints [arXiv: 1502.00791]

Diaz-Cordoves, J., Claret, A., \& Gimenez, A. 1995, A\&AS, 110, 329

di Benedetto, G. P. 1998, A\&A, 339, 858

di Benedetto, G. P. 2005, MNRAS, 357, 174

Dimitrov, W., Kolev, D., \& Schwarzenberg-Czerny, A. 2004, A\&A, 417, 689

Drimmel, R., \& Spergel, D. N. 2001, ApJ, 556, 181

Eker, Z., Bilit, S., Soydugan, F., et al. 2014, PASA, 31, 24

Gillessen, S., Eisenhauer, F., Fritz, T. K., et al. 2009, ApJ, 707, L114

González Hernández, J. I., \& Bonifacio, P. 2009, A\&A, 497, 497

Graczyk, D., Pietrzyński, G., Thompson, I. B., et al. 2014, ApJ, 780, 59

Høg, E., Fabricius, C., Makarov, V. V., et al. 2000, A\&A, 357, 367

Houdashelt, M. L., Bell, R. A., \& Sweigert, A, V. 2000, AJ, 119, 1448

Houk, N., \& Swift, C., 1999, Michigan Catalogue of Two-dimensional Spectral Types for the HD Stars Vol. 5. Declinations -12 deg to +05 deg. Department of Astronomy, University of Michigan, Ann Arbor, MI, USA

Irwin, J. B. 1959, AJ, 64, 149

Johnson, D. R. H., \& Soderblom, D. R. 1987, AJ, 93, 864

Kazarovets, A. V., Samus, N. N., Durlevich, O. V., et al. 1999, IBVS, No. 4659

Kervella, P., Thévenin, F., Di Folco, E., \& Ségransan, D. 2004, A\&A, 426, 297

Kruszewski, A., \& Semeniuk, I. 1999, Acta Astron., 49, 561

Lacy, C. H. 1977, ApJ, 213, 458

Masana, E., Jordi, C., \& Ribas, I. 2006, A\&A, 450, 735

Maxted, P. F. L., Bloemen, S., Heber, U., et al. 2014, MNRAS, 437, 1681

Mayor, M., Pepe, F., Queloz, D., et al. 2003, The Messenger, 114, 20

Munari, U., \& Zwitter, T. 1997, A\&A, 318, 269

Pecaut, M. J., \& Mamajek, E. E. 2013, ApJS, 208, 9
Perryman, M. A. C., Lindegren, L., Kovalevsky, J., et al. 1997, A\&A, 323, L49 Perryman, M. A. C., de Boer, K. S., Gilmore, G., et al. 2001, A\&A, 369, 339 Pickles, A., \& Depagne, E. 2010, PASP, 122, 1437

Pietrzyński, G., Graczyk, D., Gieren, W., et al. 2013, Nature, 495, 76

Pilecki, B., Konorski, P., \& Górski, M. 2012, From Interacting Binaries to Exoplanets, IAU Symp., 282, 301

Pojmański G., 2002, Acta Astron., 52, 397

Poleski, R. 2013, ArXiv e-prints [arXiv: 1306. 2945]

Pollacco, D. L., Skillen, I., Collier Cameron, A., et al. 2006, PASP, 118, 1407

Popper, D. M., \& Etzel, P. B. 1981, AJ, 86, 102

Ramírez, I., \& Meléndez, J. 2005, ApJ, 626, 465

Rappaport, S., Deck, K., Levine, A., et al. 2013, ApJ, 768, 33

Rucinski, S. M. 1992, AJ, 104, 1968

Rucinski, S. M. 1999, in Precise Stellar Radial Velocities, eds. J. B. Hearnshaw, \& C. D. Scarfe, IAU Colloq. 170, ASP Conf. Ser., 185, 82

Schlafly, E. F., \& Finkbeiner, D. P. 2011, ApJ, 737, 103

Schlegel, D. J., Finkbeiner, D. P., \& Davis, M. 1998, ApJ, 500, 525

Southworth, J. 2014, ArXiv e-prints [arXiv: 1411.5517]

Southworth, J., Maxted, P. F. L., \& Smalley, B. 2004a, MNRAS, 351, 1277

Southworth, J., Zucker, S., Maxted, P. F. L., \& Smalley, B. 2004b, MNRAS, 355, 986

Stebbins, J. 1910, ApJ, 32, 185

Stebbins, J. 1911, ApJ, 34, 112

Suchomska, K., Graczyk, D., Smolec, R., et al. 2015, MNRAS, 451, 5170

Tamuz, O., Mazeh, T., \& Zucker, S. 2005, MNRAS, 356, 1466

Torres, G., Andersen, J., \& Giménez, A. 2010, A\&AR, 18, 67

van Hamme, W. 1993, AJ, 106, 2096

van Hamme, W., \& Wilson, R. E. 2007, ApJ, 661, 1129

van Leeuwen, F. 2007a, HipPARcos, the new reduction of the raw data (Dordrecht: Springer)

van Leeuwen, F. 2007b, A\&A, 474, 653

Wilson, R. E. 1979, ApJ, 234, 1054

Wilson, R. E. 1990, ApJ, 356, 613

Wilson, R. E., \& Devinney, E. J. 1971, ApJ, 166, 605

Wilson D. M., Gillon, M., Hellier, C., et al. 2008, ApJ, 675, L113

Woltjer, J. 1922, B.A.N., 1, 93

Zacharias, N., Monet, D. G., Levine, S. E., et al. 2004, BAAS, 36, 1418 